\title{
Use of Point of Care-Guided Coagulation Therapy During Massive Transfusion
}

\author{
Lidia Mora Miquel, ${ }^{1, *}$ and Masood Mohseni ${ }^{2}$ \\ ${ }^{1} \mathrm{MD}$, Clinical Associate Professor, Department of Surgery, Universitat Autonoma de Barcelona, Barcelona, Spain \\ ${ }^{2} \mathrm{MD}$, Associate Professor, Department of Anesthesiology, Iran University of Medical Sciences, Tehran, Iran \\ "Corresponding author: Lidia Mora Miquel, Clinical Associate Professor, Department of Surgery, Universitat Autonoma de Barcelona, Barcelona, Spain. E-mail: \\ lmora@vhebron.net \\ Received 2017 January 25; Accepted 2017 February 28.
}

Keywords: Transfusion, Coagulation, Viscoelastic, Thromboelastography

Based on the emergency of a severely bleeding patient, both in spontaneous, traumatic, surgical or the obstetric context, the following premises are initially proposed: the haemorrhagic shock is a preventable cause of death, and the severe haemorrhage could be managed in advance when goal-directed coagulation therapy is applied at the right time with an early re-evaluation (point of care), to optimize the blood resources, save transfusion, minimize morbidity, and improve patient survival (1-3). The current demand for an optimal diagnostic tool that allows to adapt to the urgency of a bleeding adult or paediatric (4) patient that requires a fast and precise action in a life-threatening situation, is forcing to act as soon as possible and in a targeted manner.

Nowadays, viscoelastic monitoring of coagulation at bedside is used worldwide as it offers the demanded characteristics $(5,6)$ such as rapid and accurate monitoring of clot formation and destruction, early differential diagnosis of coagulopathy, individualized treatment guidelines following the decision algorithms, good reliability, and very fast response time. There are currently 2 viscoelasticbased coagulation technologies with wide clinical application and sufficient scientific evidence of their effectiveness and excellent results: thromboelastometry (ROTEM $®)$ and thromboelastography (TEG®).

The experience and scientific evidence of employing viscoelastic technologies in the patients with haemorrhagic shock positively increased in the last decade (7-9). The objective: to reduce or avoid the transfusion of blood products required to reanimate severe bleeding, without wasting them or overtransfusing the patient, saving on economic resources and acting in the most specific way possible.

The best way to reanimate a severe haemorrhage, in which the risk of coagulopathy is very high, is to consider the best resources and the easiest accessibility to them. It is essential to know the pathophysiology of the procedure that causes bleeding; for example, the acute trauma induced coagulopathy in a severely injured patient, where the viscoelastic approach is described in several studies (10-12) conducted to highlight the importance of early diagnosis of hyperfibrinolysis, since it is directly related to the increase of mortality. The gold standard to diagnose hyperfibrinolysis is viscoelastic technology. Thus, it is a fundamental tool to diagnose and treat acute traumatic coagulopathy, and also in other conditions such as liver disease (13), postpartum haemorrhage (14), or cardiac surgery (15). The current European guidelines for management of trauma bleeding patients (16) recommend the use of early and repeated monitoring of coagulation, and using a traditional laboratory test (grade $1 \mathrm{~A}$ ) and/or a viscoelastic method (grade 1C).

The broad concept of damage control resuscitation comes from the military setting in the context of the patient with trauma and advocates the management of severe haemorrhage with the application of fixed ratios of transfusion 1:1:1, with fresh frozen plasma as main resuscitation fluid. But even with the last randomized control trial with high scientific evidence (the pragmatic randomized optimal platelet and plasma ratios (PROPPR) Study (17)), a totally favourable conclusion about the use of this approach could not be fully obtained, when trying to compare 1:1:1 to 1:1:2 approaches.

In common clinical practice, depending on the blood products and prothrombotic available drugs it can be acted following the haemostatic protocols and it is always a matter of time and velocity to the physician who leads the resuscitation. There are circumstances in which it is necessary to transfuse very quickly and with great volume, but at the moment when a blood sample can be extracted and analysed in a viscoelastic method, the therapeutic guide marked by the proof of concept (POC) technology is very 
useful, as the therapeutic efforts are directed to replace only those haemostatic deficient components. In severe active bleedings, the standard coagulation tests are too slow to take fast clinical judgements (18). In addition, the results obtained from viscoelastic methods are validated in different studies (19).

The fundamental principle of handling these techniques is that decisions of transfusion should not be strictly made because the test results are outside a numerical range of each parameter defined by the different devices. Decisions will always be taken according to the clinical situation of the patient and according to the availability of treatment options $(20,21)$.

The therapeutic decision algorithm based on POC devices should be coupled to the massive transfusion protocol approved in each hospital $(22,23)$.The combination of these 2 procedures is what actually brings logic to the resuscitation of a haemorrhagic shock and allows taking advantage of both resources. The transfusion protocol should develop an actuation procedure for the rapid and organized dispensing of blood products from the blood bank. The combination of resources should not be observed as contradictory or incapable of being overlaid on organizational issues. It is an intense multi-disciplinary task that requires an extensive preliminary work of communication so that the protocol of action is consensual and following an ideological order. For this purpose, all medical services involved should participate in the implementation. The development of a protocol that structures the dispensation of hematic products is essential to ensure immediate action if it is necessary in the emergent cases, but it should be followed absolutely by a POC-based treatment algorithm. It requires, as already mentioned, an exhaustive teamwork.

It is certainly difficult to apply the adult-based massive bleeding protocols to children, because the traditional definition of massive transfusion cannot be considered for the paediatric patients. Therefore, estimating blood loss in $\mathrm{mL} / \mathrm{kg}$ or total blood volume in children should be considered instead of unit counts when considering massive transfusion or bleeding. Adult protocols should be adapted to the paediatric patient, not applying the ratiodriven formulas, but the POC algorithms above all, leaving aside the conventional coagulation tests for an accurate and fast resuscitation (24). It is assumed that using an algorithm based on fixed ratios of transfusion in children is also the failure of the strategy and there is not sufficient evidence to support this approach at all.

A recent Cochrane revision assessed the benefits and harms of thromboelastography or thromboelastometryguided transfusion in adults and children with severe bleeding (4). They looked at different outcomes, such as overall mortality and bleeding events, with subgroups and sensitivity analyses, also examining the role of bias to consider the evidence. The study concluded that there is growing evidence that application of POC-guided transfusion strategies may reduce the need for blood products and improve survival in patients with severe bleeding. However, these results are primarily based on trials of elective cardiac surgery involving cardiopulmonary bypass and the level of evidence remains low. Therefore, further evaluation of POC-guided transfusion in acute settings in low risk bias studies is needed.

Several reports suggest that the cost of blood products may rise over time (25). In the United States, the cost of erythrocyte transfusion exceeds 1.3 billion dollars annually (26). In the developing countries with limited financial resources, the economic burden of transfusion is even larger due to poorly settled hemovigilance systems. In transfusion medicine, several cost-reduction strategies are introduced (27). It is suggested that POC-guided transfusion may provide the opportunity to improve quality of care, while reducing the cost thereof. A review study revealed that point-of-care viscoelastic testing is cost-saving and results in reduced amounts of administered blood products without changing the clinical outcome of patients (3). Another recent survey in patients undergoing cardiac surgery suggested that incorporating viscoelastic testing into transfusion algorithms improved medical outcomes in the presented health economic model, in comparison with standard laboratory tests; despite the higher costs of testing (28). Optimizing blood usage finally improves the outcome through channelling resources toward other diagnostic and therapeutic initiatives.

Nowadays, there is a wide clinical experience in many medical-surgical disciplines to use POC-guided transfusion, even though the degree of evidence is not strictly desirable to make a unanimous and universal recommendation. For this reason, it should be mandatory for all centres where severe acute haemorrhage is attended, to implement POC technology to develop decision algorithms for haemostatic resuscitation. This could lead to the development of new randomized multicentre clinical trials that reinforce the evidence on the reduction of transfusion of blood components and, above all, the increase in survival of the patients with severe bleeding, both in adults and children.

Once the physician attending the resuscitation of a severe bleeding acquires the routine of combining the initial (maybe empirical, but not necessarily ratio-driven approach) performance with the guidelines of treatment based on point of care haemostatic technology, the results begin to improve, and the resources get optimized $(29,30)$. Viscoelastic methods provide a rapid assessment of coag- 
ulation to support clinical decision-making, generating a growing confidence in these methods, and increased use. By this technology, the general impression is that patients are treated much more specifically, accurately, and costeffectively with improved survival.

\section{References}

1. Johansson PI, Stensballe J, Oliveri R, Wade CE, Ostrowski SR, Holcomb JB. How I treat patients with massive hemorrhage. Blood. 2014;124(20):3052-8. doi: 10.1182/blood-2014-05-575340. [PubMed: 25293771].

2. Kozek-Langenecker SA. Perioperative coagulation monitoring. Best Pract Res Clin Anaesthesiol. 2010;24(1):27-40. [PubMed: 20402168].

3. Whiting P, Al M, Westwood M, Ramos IC, Ryder S, Armstrong N, et al. Viscoelastic point-of-care testing to assist with the diagnosis, management and monitoring of haemostasis: a systematic review and cost-effectiveness analysis. Health Technol Assess. 2015;19(58):1-228. doi: 10.3310/hta19580. [PubMed: 26215747] v-vi.

4. Wikkelso A, Wetterslev J, Moller AM, Afshari A. Thromboelastography (TEG) or thromboelastometry (ROTEM) to monitor haemostatic treatment versus usual care in adults or children with bleeding. Cochrane Database Syst Rev. 2016(8):CD007871. doi: 10.1002/14651858.CD007871.pub3. [PubMed: 27552162].

5. Bolliger D, Seeberger MD, Tanaka KA. Principles and practice of thromboelastography in clinical coagulation management and transfusion practice. Transfus Med Rev. 2012;26(1):1-13. doi: 10.1016/j.tmrv.2011.07.005. [PubMed: 21872428].

6. Gorlinger K, Dirkmann D, Solomon C, Hanke AA. Fast interpretation of thromboelastometry in non-cardiac surgery: reliability in patients with hypo-, normo-, and hypercoagulability. Br J Anaesth. 2013;110(2):222-30. doi: 10.1093/bja/aes374. [PubMed: 23112213].

7. Haas T, Gorlinger K, Grassetto A, Agostini V, Simioni P, Nardi G, et al. Thromboelastometry for guiding bleeding management of the critically ill patient: a systematic review of the literature. Minerva Anestesiol. 2014;80(12):1320-35. [PubMed: 24518216].

8. Hans GA, Besser MW. The place of viscoelastic testing in clinical practice. Br J Haematol. 2016;173(1):37-48. doi: 10.1111/bjh.13930. [PubMed: 26818064]

9. Wikkelso A, Wetterslev J, Moller AM, Afshari A. Thromboelastography (TEG) or rotational thromboelastometry (ROTEM) to monitor haemostatic treatment in bleeding patients: a systematic review with metaanalysis and trial sequential analysis. Anaesthesia. 2017;72(4):519-31. doi: 10.1111/anae.13765. [PubMed: 28052313].

10. Veigas PV, Callum J, Rizoli S, Nascimento B, da Luz LT. A systematic review on the rotational thrombelastometry $(\operatorname{ROTEM}(\mathrm{R}))$ values for the diagnosis of coagulopathy, prediction and guidance of blood transfusion and prediction of mortality in trauma patients. Scand $J$ Trauma Resusc Emerg Med. 2016;24(1):114. doi: 10.1186/s13049-016-03082. [PubMed: 27716278].

11. Schochl H, Voelckel W, Schlimp CJ. Management of traumatic haemorrhage-the European perspective. Anaesthesia. 2015;70 Suppl 1:102-7. doi: 10.1111/anae.12901. [PubMed: 25440403] e35-7.

12. Stensballe J, Ostrowski SR, Johansson PI. Haemostatic resuscitation in trauma: the next generation. Curr Opin Crit Care. 2016;22(6):591-7. doi: 10.1097/MCC.0000000000000359. [PubMed: 27811561].

13. Tafur LA, Taura P, Blasi A, Beltran J, Martinez-Palli G, Balust J, et al. Rotation thromboelastometry velocity curve predicts blood loss during liver transplantation. Br J Anaesth. 2016;117(6):741-8. doi: 10.1093/bja/aew344. [PubMed: 27956672].
14. Le Gouez A, Mercier FJ. Major obstetric hemorrhage. Transfus Clin Biol. 2016;23(4):229-32. doi: 10.1016/j.tracli.2016.08.003. [PubMed: 27592155].

15. Besser MW, Ortmann E, Klein AA. Haemostatic management of cardiac surgical haemorrhage. Anaesthesia. 2015;70 Suppl 1:87-95. doi: 10.1111/anae.12898. [PubMed: 25440401] e29-31.

16. Rossaint R, Bouillon B, Cerny V, Coats TJ, Duranteau J, FernandezMondejar E, et al. The European guideline on management of major bleeding and coagulopathy following trauma: fourth edition. Crit Care. 2016;20:100. doi: 10.1186/s13054-016-1265-x. [PubMed: 27072503].

17. Holcomb JB, Tilley BC, Baraniuk S, Fox EE, Wade CE, Podbielski JM, et al. Transfusion of plasma, platelets, and red blood cells in a 1:1:1 vs a 1:1:2 ratio and mortality in patients with severe trauma: the PROPPR randomized clinical trial. JAMA. 2015;313(5):471-82. doi: 10.1001/jama.2015.12. [PubMed: 25647203].

18. Haas T, Fries D, Tanaka KA, Asmis L, Curry NS, Schochl H. Usefulness of standard plasma coagulation tests in the management of perioperative coagulopathic bleeding: is there any evidence? Br J Anaesth. 2015;114(2):217-24. doi: 10.1093/bja/aeu303. [PubMed: 25204698].

19. Solomon C, Asmis LM, Spahn DR. Is viscoelastic coagulation monitoring with ROTEM or TEG validated? Scand J Clin Lab Invest. 2016;76(6):503-7. doi: 10.1080/00365513.2016.1200136. [PubMed: 27354213].

20. Theusinger OM, Stein P, Levy JH. Point of care and factor concentratebased coagulation algorithms. Transfus Med Hemother. 2015;42(2):11521. doi: 10.1159/000381320. [PubMed: 26019707].

21. Meesters MI, Koning NJ, Romijn JWA, Loer SA, Boer C. Clinical decision versus thromboelastometry based fresh frozen plasma transfusion in cardiac surgery. BrJAnaesth. 2017;118(3):458-9. doi:10.1093/bja/aex011. [PubMed: 28203761].

22. Stensballe J, Ostrowski SR, Johansson PI. Viscoelastic guidance of resuscitation. Curr Opin Anaesthesiol. 2014;27(2):212-8. doi: 10.1097/ACO.0000000000000051. [PubMed: 24514038].

23. Mehaffey JH, Schubert SA, Gelvin MG, Charles EJ, Hawkins RB, Johnston LE, et al. A New Intraoperative Protocol for Reducing Perioperative Transfusions in Cardiac Surgery. Ann Thorac Surg. 2017 doi: 10.1016/j.athoracsur.2016.10.032. [PubMed: 28131428].

24. Haas T, Spielmann N, Mauch J, Madjdpour C, Speer O, Schmugge $\mathrm{M}$, et al. Comparison of thromboelastometry (ROTEM(R)) with standard plasmatic coagulation testing in paediatric surgery. BrJAnaesth. 2012;108(1):36-41. doi: 10.1093/bja/aer342. [PubMed: 22086509].

25. Toner RW, Pizzi L, Leas B, Ballas SK, Quigley A, Goldfarb NI. Costs to hospitals of acquiring and processing blood in the US: a survey of hospital-based blood banks and transfusion services. Appl Health Econ Health Policy. 2011;9(1):29-37. doi: 10.2165/11530740-00000000000000. [PubMed: 21174480].

26. Szpisjak DF, Potter PS, Capehart BP. Economic analysis of an intraoperative cell salvage service. Anesth Analg. 2004;98(1):201-5. [PubMed: 14693619] table of contents.

27. Petrides M. When less is more: cost-containment in transfusion medicine. Transfus Sci. 1997;18(4):603-12. [PubMed: 10178686].

28. Straub N, Bauer E, Agarwal S, Meybohm P, Zacharowski K, Hanke AA, et al. Cost-Effectiveness of POC Coagulation Testing Using Multiple Electrode Aggregometry. Clin Lab. 2016;62(6):1167-78. [PubMed: 27468580].

29. Williams B, McNeil J, Crabbe A, Tanaka KA. Practical Use of Thromboelastometry in the Management of Perioperative Coagulopathy and Bleeding. Transfus Med Rev. 2017;31(1):11-25. doi: 10.1016/j.tmrv.2016.08.005. [PubMed: 27622549].

30. Gorlinger K, Fries D, Dirkmann D, Weber CF, Hanke AA, Schochl H. Reduction of Fresh Frozen Plasma Requirements by Perioperative Point-of-Care Coagulation Management with Early Calculated GoalDirected Therapy. Transfus Med Hemother. 2012;39(2):104-13. [PubMed: 22670128]. 